\title{
Research on the Representation of Line- type of Digital Sketching in Product Design
}

\author{
Liu Ying ${ }^{1}$, Liu Xiaojing ${ }^{1}$ and Chen $\mathrm{Yi}^{2}$, a \\ ${ }^{1}$ Cnetral South University, Industrial Design Department, 410083 Changsha Hunan, China \\ ${ }^{2}$ Guzhi Science and Technology Co., Ltd. 410083 Changsha Hunan, China
}

\begin{abstract}
The type of lines which from digital hand drawing representation is the central element to the constituted form. At present, there are a few special topics on the structural description of the type of lines. According to the classification of kind of lines which from the basis of painting, integrated using of various lines can create spatial illusions in the plane horizon to achieve the performance of three-dimensional entities. By clarifying features and characteristics of various lines, the digital technology can be quickly generated to design the representation effect and form the graphic design language.
\end{abstract}

\section{Introduction}

Digital hand drawing is not only synthesizes the effect characteristics of many drawing tools but also simulates the effect of paper hand drawing to realise paperless painting. The stored record function of the computer can also support the repeated modification of pictures, which is a kind of a closer expression means to combine technology and art ${ }^{[1]}$. The performance features which is fast and convenient can provide technical support for design representation and communication. At present, the research on hand drawing design is focused on the techniques of skill which from marker pen and the teaching curriculum system, while there are a few special topics for product structure representation ${ }^{[2-4]}$. The core of product form design is the continuation and reconfiguration of the form ${ }^{[5]}$, so the structure analysis and structure of the type of lines are the research focus in digital design. The research of kind of lines classifies the function of the line, conducts differential effect processing in the picture, synthesises technical characteristic of digital representation, integrates the technical characteristics of the digital image to form the spatial perspective and designs diagram of the shape structure.

\section{Classification of lines}

Line is the soul of hand drawing representation ${ }^{[6]}$. As the design representation under the background of emerging technology, digital hand drawing is a technical extension derived which from the basis of structural sketch line. Highly generalised lines are needed by the plotter to describe the physical characteristics of the object. Objects can be presented intuitively in real space, but lines are not real, they are the space boundary between two objects or between object and environment under the condition of light. Spatial boundary of the object is formed by the reflection of light where on the boundary of object and

the environment, which is commonly referred to as the "contour line"; the reflection of the light between the object's own surface makes a difference in intensity, thus forming "light and dark line" which can be perceived by vision in objective. The die line is the overlapping edge which is caused by the need for production and splicing of blocks and surfaces, which is also the spatial boundary between each component. These three lines can be defined as solid lines by visual perception, that means, visual lines that actually exist on real objects.

\subsection{Functional classification of lines}

Several types of lines which are used in digital hand drawing cannot be visually seen. In order to describe characteristics of objects in detail, designers can use a large number of auxiliary lines, which are based on the cognitive framework of spatial thinking. Because of their difference in functional utility, they can be divided into structural lines, perspective lines, analytical lines, cross section lines, etc.

As shown in figure 1, the structural line is the characteristic line which used by the designer to describe the shape of objects. Being the structural component that determines the shape of the object, which can reflect the turning point, span an extension of the surface. The structure line is fixed compared to the object, and it does not move with the angle of perspective. The perspective line is used to assist the generation of spatial meaning, which is usually accompanied by indicator arrows to create visual direction and guide fabricate threedimensional space in visual perception. In order to better explaining the structure and shape of the product, the

*Corresponding author: ${ }^{\text {a } 48316365 @ q q . c o m}$ 
cross-section line is a boundary formed by the designer's hypothetical cutting through the object, which can be used to explain the shape and structure of the object.

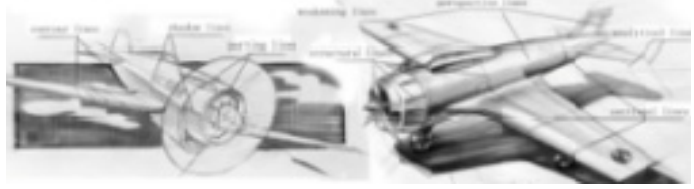

Figure 1. solid and dotted lines

All types of lines (as shown in Form 1) are comprehensively used in drawing representation and are not isolated. The description of the features of solid and dotted lines is not an absolute definition. The outline of an object usually refers to the continuous edge of a structural line under a fixed angle of view, and the profile line is often described as solid lines which is the outline in product decomposition map. The strength and weakness of types of lines should be treated with a specific situation. Virtual-actual transformation of visual perception is produced by differentiation of straight curve, thickness, softness and hardness, speed and weight of picture.

Form 1. Linear classification

\begin{tabular}{|c|c|c|c|c|c|c|}
\hline \multicolumn{3}{|c|}{ Solid lines } & \multicolumn{4}{|c|}{ Dotted lines } \\
\hline $\begin{array}{l}\text { cont } \\
\text { our } \\
\text { lines }\end{array}$ & $\begin{array}{l}\text { outli } \\
\text { nes }\end{array}$ & $\begin{array}{l}\text { light } \\
\text { and } \\
\text { dark } \\
\text { line }\end{array}$ & $\begin{array}{l}\text { cross } \\
- \\
\text { secti } \\
\text { on } \\
\text { line }\end{array}$ & $\begin{array}{l}\text { struct } \\
\text { ural } \\
\text { line }\end{array}$ & $\begin{array}{l}\text { perspe } \\
\text { ctive } \\
\text { line }\end{array}$ & $\begin{array}{l}\text { ana } \\
\text { lyti } \\
\text { cal } \\
\text { line }\end{array}$ \\
\hline \multicolumn{2}{|c|}{$\begin{array}{l}\text { Emphasis on } \\
\text { treatment }\end{array}$} & $\begin{array}{l}\text { Gener } \\
\text { al } \\
\text { treat } \\
\text { ment }\end{array}$ & \multicolumn{2}{|c|}{$\begin{array}{l}\text { Weakening } \\
\text { treatment }\end{array}$} & \multicolumn{2}{|c|}{$\begin{array}{l}\text { Weakest } \\
\text { treatment }\end{array}$} \\
\hline
\end{tabular}

\subsection{Differentiation of lines}

According to the function of their pictures, the virtualactual transformation of visual perception is produced by differentiation of straight curve, thickness, softness and hardness, speed and weight. Digital hand drawing inherits the line feature of structure sketch, and the representation of a line in picture is not only a single form expression. The computer can provide pressuresensing function. The plotter only needs to emphasize the virtual-actual linear change through the difference of hand movement, and make full use of the thickness, weight, and extension of lines to create spatial illusions in near and far space in the plane horizon, thus achieving performance of three-dimensional entity. As shown in figure 2 , the cube at perspective angle forms the contour lines with nine solid lines. In order to create the visual illusion in the far and near space, the contour lines should be drawn apart from the visual differences: line A from the nearest visual point has the heaviest color, then is line $\mathrm{B}$ and $\mathrm{C}$, the contour line of the visual bottom is slightly influenced by projection, and the line D and $\mathrm{E}$ increases the width; visual level of the remote contour is on the back, the line $\mathrm{G}$ and I needs to be weakened in thickness and color; and longitudinal and horizontal structure of the object surface is used as the auxiliary line to further weaken the strength.
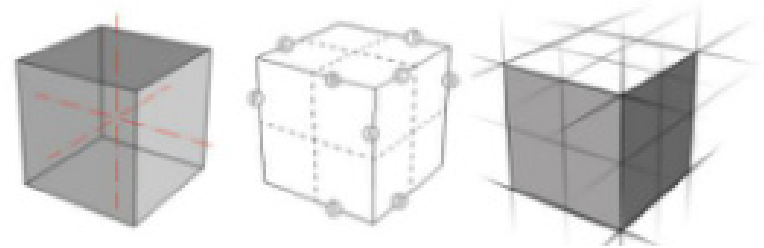

Figure2. Linear differential representation

Vision is a basic tool for thinking [7]. At the initial creation of the draft, the designer should always quickly to record the sporadic, fleet design inspiration, turn the vague intention into a visual representation and regard it as the original material for the idea. Compared with the rigorous perspective chart, the fast-recording drawing speed will increase the random degree of type of lines. However, in this painting stage, the representation of auxiliary line is still essential. Figure 3 shows a quick representation diagram in which we can see impromptu interest and certain design gesture ${ }^{[8]}$. There are a large number of structural lines that represent the bending of the body, cross section lines that indicate the variation of the area and perspective lines to assist space coordinate. The comprehensive application of multiple lines can enrich the structure of the picture and carry more structural design information so as to enhance communication and discussion of design.

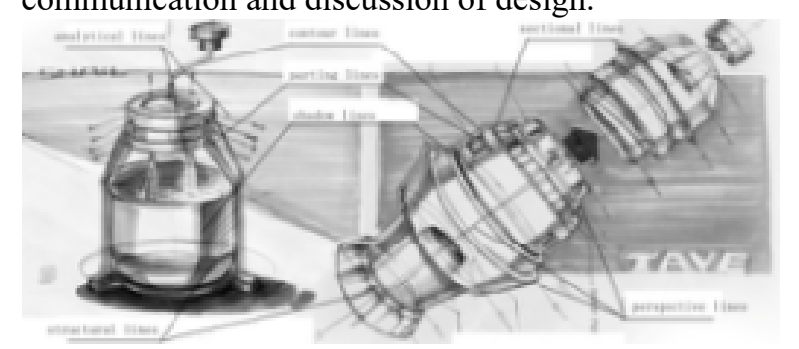

Figure 3. Application of type of lines in fast representation

\subsection{Application of type of lines in comprehensive performance}

When digital hand drawing is used to express the design effect, the line is the main representation factor of the form, and the structure mode of the design object plays a decisive role in the requirement of type of lines. The thickness, length, width, twists and turns of lines depend on the structure trend of the shape of the object. The technological advantage of the digital representation makes the line more fluent, directive and hierarchic, which makes designers easy to grasp the characteristics of line; solid lines are used to describe visual contour, while dotted lines assist the generation of spatial structure. As shown in Figure 4, lines are used to establish the basic structural characteristics of the form. In the representation of spatial effect, the digital technology can give full play to the technological advantages of the computer and help teachers and students to set up the structure quickly. The methods and technique of graphic language are used to express, communicate and evaluate various design information 
and design plans, as well as to transform creative ideas into graphic design language ${ }^{[9]}$.

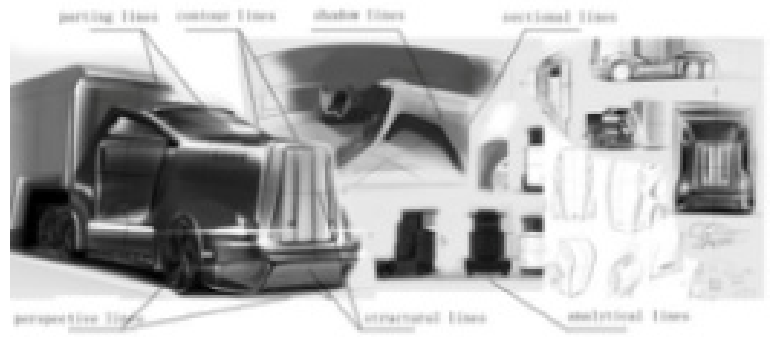

Figure 4. Application of type of lines in comprehensive representation

\section{Conclusion}

Digital hand drawing focuses on structural analysis and line formation. The designer should first distinguish the functional types of lines in the screen, then use the differential representation form to create visual level and make space effect, and finally to describe the structure composition and surface features of the design objects in detail through various auxiliary type of lines. The design drawings with distinct effect can highlight the key points of design, enhance visual language of the picture and enrich design information of drawings. In order to clarify difference of type of lines and analyze the modeling structure in detail, sustainable development of digital representation technology will provide more possibility for type of lines and synthetic technique.

\section{Acknowledgment}

The paper is the final supporting material for Hunan philosophy and social science foundation project "Industrial design value and performance evaluation research", Item No.: 16yba369

\section{References}

1. Xue Yanmin, Deng Binshuo, Wang Jiamin. The position and function of fast representation of hand drawing in product Design. Packaging Engineering, 08(2008)

2. Ren Min, Wu Lili. Creative performance of hand drawing products under the background of cultivating applied talents. Art Education Research ,19(2017)

3. Wang Xueqin, Zheng Yinfeng. Teaching of representation techniques of hand drawing for products. Mass literature and art, 03 (2017)

4. Liu Yang, Zhang Zhiming, Xia Lili. The representation technique of hand drawing. Beijing Arts and Crafts Press.( 2016)

5. Chen Zhenyi. Analysis and reconstructionexploration of the theory of typology in teaching of furniture improvement design in Ming and Qing dynasties. Adornment ,04(2016)
6. Li Yinxing. Discussion on the training model of students'ability in product hand drawing. Adornment, 09(2013)

7. Arnheim $\cdot$ Rudolph. Visual thinking. translated by Teng Shouyao, Guangming Daily Press. (1986)

8. Zhong Xuhui, $\mathrm{Xu}$ Lei. The role of hand drawing in teaching of ring art design. Packaging Engineering ,26(2005)

9. An Jingbin. The function of hand drawing in product design process. Industrial design 01(2017) 